\title{
EDITORIAL
}

\section{Occupational exposure to magnetic fields and brain cancer}

The potential carcinogenicity of electric and magnetic fields encountered in "electrical occupations" has been of concern to researchers and the public for nearly 20 years. Increasingly complex methods of occupational epidemiology have been applied to this issue, culminating in a series of reports in the past decade from studies that are large, apply intricate methods of exposure assessment, and use complex statistical analyses. The article by Sorahan et al examining mortality from brain cancer among over 80000 employees of the former Central Electricity Generating Board is a methodologically strong addition to these publications.

As was found among southern Californian Edison workers in the United States ${ }^{2}$ and Danish electric utility workers, ${ }^{3}$ the British workers show no indications whatsoever of an association between measures of exposure to magnetic fields and mortality from brain cancer. By contrast, the study of French and Canadian utility workers provided sporadic positive findings for astrocytomas and benign tumours, ${ }^{4}$ with a suggestion of electric fields conferring increased risk among the French workers in that study. ${ }^{5}$ The cohort of several United States electric utility company employees yielded a clear positive association between exposure to magnetic fields and brain cancer, including a dose-response gradient, particularly for recent exposure. ${ }^{6}$ In the light of the cumulative body of research, including this most recent report on electric utility workers and brain cancer, it is appropriate to ask where we stand on this topic and where we are going.

To help assess the current state of knowledge about cancer among electric utility workers, we recently undertook a coordinated analysis of three of the largest studies ${ }^{24}{ }^{4}$ to examine differences in methods and attempt to reconcile results. $^{7}$ The many fine points of methodology, including inclusion criteria, exposure categorisation, handling of potential confounding factors, and specific analytical approaches, each could make a difference in the reported findings. In trying to reconcile findings among reasonably well conceived and properly executed and analyzed studies, such subtleties will rarely make a major difference to the results. The exposure assessment strategy of Sorahan et $^{1} \mathrm{l}^{1}$ and several details of the analysis are different from those used in the previous studies (which differ from one another) but it is doubtful whether the flatly negative results would change through small modifications to the methods. Weighting the new study results with those that preceded it, the very small increase in risk suggested for brain cancer found in the pooled analysis (relative risk of 1.1 per 10 microTesla-years of exposure), already below the range for meaningful epidemiological inference, would be smaller still.

The most striking aspect of the coordinated analysis was the degree to which the studies with findings from large studies that were ostensibly contradictory were statistically compatible with one another, taking random error into account. All (including the recent study) are compatible with a very small increased risk of brain cancer associated with increasing exposure to magnetic fields. The positive findings reported for United States utility workers ${ }^{6}$ could well result from some insidious combination of as yet unexplained methodological problems and random error. It is equally plausible that unidentified systematic and random errors could have caused the study by Sorahan et al to miss a true, small increased risk.

Our current failure to be able to explain and reconcile discrepant findings based on structural features of the studies is troubling. However, one of the central arguments for the value of replication is the opportunity to consider both known methodological deficiencies and unknown sources of error in research findings, including random error. At least for electric utility workers, and quite possibly for occupational electric and magnetic fields more generally (at exposure levels that can be examined in very large cohorts), we seem to have a fairly complete answer for brain cancer. We may well be doing a disservice not to share the good news more energetically and widelyelectric utility workers and other similar such workers do not seem to be at measurably increased risk of brain cancer.

As researchers, it might seem heretical not to argue that "further study is needed." But we need to consider more carefully why it is needed and outline strategies that are likely to yield important new information. More studies of the general nature of the United Kingdom utility worker study are not needed, given the inability of such research to change appreciably the conclusion just reached. If a new study were to shift the combined estimate of the relative risk per microTesla-year from 1.10 to 1.05 or 1.15 per 10 microTesla-years, it would hardly be worth the trouble for regulatory decision making or for advancing the science. Perhaps if there were some biological insight that pointed to a "magnetotoxicant," and a large group of workers with the presumed potent exposure could be isolated for study, then epidemiological inquiry on associated risks for brain cancer would be warranted as strikingly positive findings could markedly alter the cumulative evidence. However, the burden of proof is on those who claim to have discovered the "magnetotoxicant." They would need to make the case that the harmful exposure has been missed in previous evaluations of time weighted average and cumulative exposure indices used in past studies.

Alternatively, if a uniquely exposed population were identified, or a notably better method of reconstructing historical exposure were found-for example, an archive that included decades of the equivalent of film badges for ionising radiation or a biological marker of integrated exposure-then there would be the potential to overturn the largely negative studies that all have substantial exposure misclassification. For now, however, on the restricted topic of typical time weighted average occupational exposure to electric and magnetic fields and brain cancer, further study of the type done thus far is not needed. This conclusion does not, however, apply to research on residential exposure; nor does it argue against the potential value of studies of occupational exposure to electric and magnetic fields relative to less extensively investigated health end points, such as breast cancer or neurodegenerative disease. 
I acknowledge the review and constructive comments by Drs Harvey Checkoway and Dana Loomis, and Mr Edwin van Wijngaarden.

D A SAVITZ

Department of Epidemiology, University of North Carolina School of Public Health, Chapel Hill, North Carolina 27599-7400, USA

david_savitz@unc.edu

1 Sorahan T, Nichols L, van Tongeren $\mathrm{M}$, et al. Occupational exposure to magnetic fields in relation to mortality from brain tumours: updated and revised findings from a study of United Kingdom electricity generation and transmission workers, 1973-97. Occup Environ Med 2001;58:626-30.

2 Sahl JD, Kelsh MA, Greenland S. Cohort and nested case-control studies of hemopoietic cancer and brain cancer among electric utility workers. Epidemiology 1993;4:104-14.
3 Johansen C, Olsen JH. Risk of cancer among Danish utility workers: a nationwide cohort study. Am f Epidemiol 1998;147:548-55.

4 Thériault G, Goldberg $M$, Miller AB, et al. Cancer risks associated with Theriault $\mathrm{G}$, Goldberg $M$, Miller AB, et al. Cancer risks associated with
occupational exposure to magnetic fields among electric utility workers in Ontario and Quebec, Canada, and France: 1970-89. Am f Epidemiol 1994; 139:550-72.

5 Guénel P, Nicolau J, Imbernon E, et al. Exposure to 50-Hz electric field and incidence of leukemia, brain tumors, and other cancers among French electric utility workers. Am f Epidemiol 1996;144:1107-21.

6 Savitz DA, Loomis DP. Magnetic field exposure in relation to leukemia and brain cancer mortality among electric utility workers. Am f Epidemiol 1995; 141:123-34.

7 Kheifets LI, Gilbert ES, Sussman SS, et al. Comparative analyses of the studies of magnetic fields and cancer in electric utility workers: studies from

France, Canada, and the United States. Occup Environ Med 1999:56:56774.

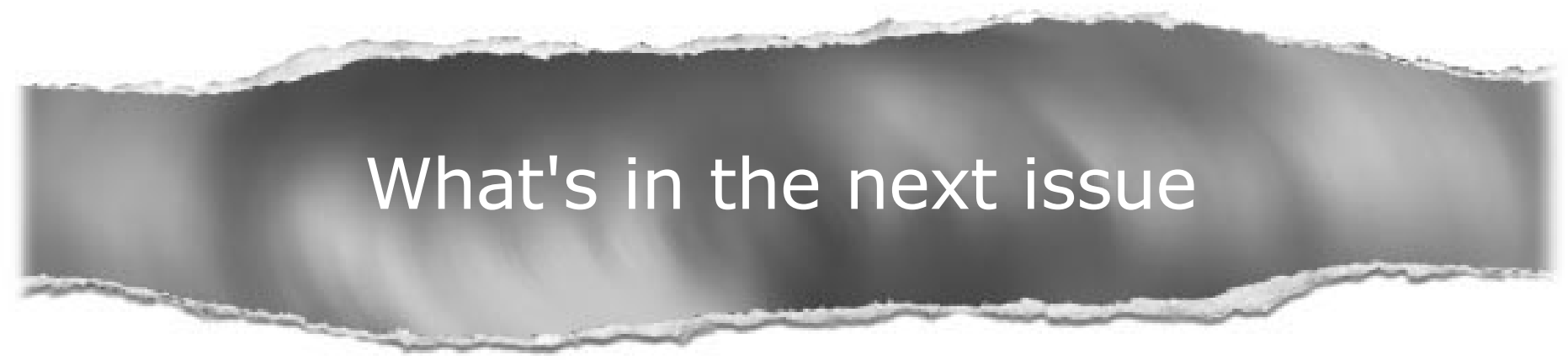

Future content

See which articles have just been accepted for publication and preview the table of contents for the next issue a month before it is published

www.occenvmed.com 